\title{
Re-emergence of the knotty chikungunya virus: facts, fear or fiction
}

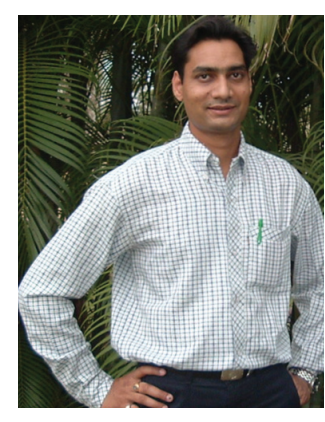

Shailendra K Saxena Centre for Cellular \& Molecular Biology, Uppal Road, Hyderabad 500 007 (AP), India Tel.: +914027192630; Fax: +914027160591 ; shailen@ccmb.res.in future, part of fso

\section{'The situation suggests \\ that despite infecting millions of people worldwide, the search for any antiviral against CHIKV has been neglected.'}

Recent large-scale outbreaks of fever caused by chikungunya virus (CHIKV) infection in several parts of the world have confirmed the re-emergence of this virus. Its vast and rapid spread is a warning for future preparedness against emerging viruses around the globe. There is no specific medicine and vaccine against the infection, therefore it is important to assess the current status and unique features of the chikungunya infection (Box 1).

\section{Etymology}

The name 'chikungunya' means 'that which bends up' in Makonde language, after the disease was first identified on the Makonde plateau, along the border between Tanzania and Mozambique, in 1952.

\section{Chikungunya ramifications: implications} of the return of the virus

The recent CHIKV epidemic that swept through the idyllic islands of the Indian Ocean marked the resurgence of CHIKV, reaching a quasi-pandemic level. According to the WHO, two-thirds of the world population lives in tropical areas where the vector for CHIKV is widely distributed. So all are at potential risk of infection. CHIKV is enzootic in various countries of Asia and Africa. During the 1960s and 1970s, CHIKV outbreaks were common in its endemic areas, Africa and SouthEast Asia, including India [1]. The recent outbreaks in Africa and Asia were unanticipated, with an interval of 7-20 years.

The most recent outbreak of CHIKV, in 2004, started in Kenya, Africa, where CHIKV is endemic and has a forest reservoir in wild primates and their vector mosquitoes. It then spread to the Comoros Islands early in 2005 and later to Mayotte, the Seychelles, Réunion and Mauritius. Since then, several hundred thousand cases have been reported in these islands. CHIKV developed exponentially at the beginning of 2006, during the southern summer. Later, circulation of the virus was also reported in Madagascar, India, Malaysia, Indonesia and Sri Lanka, where the epidemic erupted with a much larger magnitude. The re-emergence is of unprecedented magnitude and has occurred after an interval of several years in most of the affected countries. More than 1.6 million suspected cases have been reported from Réunion, Mayotte, the Seychelles, Comoros, Madagascar, Mauritius, India and Malaysia. In India reported infection rates reached $45 \%$ in some areas. Widespread occurrence of disease caused substantial morbidity and economic loss.

\section{Major observations in the recent Chikungunya epidemic \& quasi-pandemic Virus: the knotty agent}

CHIKV is an enveloped icosahedral virus with a $70 \mathrm{~nm}$ diameter, classified in the Alphavirus genus of the family of Togaviridae. The CHIKV genome is a monopartite, 5' capped, $3^{\prime}$ polyadenylated, positive-sense, approximately $11.8 \mathrm{~kb}$ long, single-stranded RNA. There are two open reading frames in the genome encoding for nonstructural and structural proteins.

'...the epidemic strain may have resulted from the evolution of an enzootic strain.'

The virus responsible for the current epidemic is similar to East, Central and South-African isolate genotypes of CHIKV. Schuffenecker and colleagues found the outbreak strain to be new and speculated that the epidemic strain may have resulted from the evolution of an enzootic strain [2]. Similar kinds of studies carried out in India, Malaysia and Hong Kong suggested that a similar strain was also circulating in these areas. Sequencing of the E1 region of the viral genome revealed interesting findings regarding the possible cause behind the re-emergence of CHIKV. The new isolate has an A226V mutation in the membrane fusion glycoprotein E1. A similar genetic change may have made the Semiliki Forest Virus, a close relative of CHIKV, independent of cholesterol, which is often lacking in mosquitoes. Thus, the newly emerged isolate with the mutation 


\section{Box 1. Summary points: chikungunya at a glance.}

- Chikungunya is an arboviral illness caused by the chikungunya virus (CHIKV) and transmitted by Aedes mosquitoes

- The disease is prevalent in the countries of Africa and South East Asia and, since 2005, has emerged in the Indian Ocean (Réunion, Mauritius and Comoros) and India

- Within the last 40 years, the virus caused the largest outbreaks of chikungunya in 2005-2006

- A single isolate, which probably originated in Africa, swept across almost all tropical countries and was exported to nonendemic areas (Europe and USA) by travellers

- CHIKV infection presents with high fever, severe arthralgia and a rash lasting for a period of 1-7 days

- Severe manifestation may cause benign hemorrhages (epistaxis and gingivorrhagia), especially in children

- CHIKV has infected millions of people worldwide and killed 200 people in Réunion

- No antiviral drugs or vaccines specific to chikungunya are available to date

might have adapted to survive better in mosquitoes, which, in turn, could have contributed to its rapid spread. However, in this context, it would be worthwhile to reconfirm this hypothesis, as well as the genotypes of currently circulating strains in South East Asia, modes of transportation of this strain in various countries, and the conditions that favored such a quasi-pandemic situation.

\section{Deadly communication of reservoir, vector \& host}

Reservoir

The common reservoirs for CHIKV are monkeys and other vertebrates, which maintain CHIKV. In the recent quasi-pandemic situation, suspected reservoirs were nonhuman primates (baboons, vervet monkeys, macaque monkeys and lemurs) and also bald mice. The role of cattle and rodents has also been reported in earlier outbreaks.

\section{CHIKV transporter: common vector, uncommon cargo}

The disease is transmitted by the mosquitoes of the subgenera Diceromyia, Stegomyia and Aedimorphus. Through a feral transmission cycle, the virus is maintained between mosquitoes and nonhuman primates, which may further aid transmission to humans. In the current outbreak, suspected vectors were Aedes aegypti, Aedes albopictus, Aedes luteocephalus, Aedes furcifer and Aedes taylori. However, a sufficiently high population density of A. albopictus and A. aegypti in tropical regions spread CHIKV over large areas of Africa and Asia.
A. albopictus, the species implicated in the Réunion outbreak, is of Asian origin and was first described by Skuse in 1894 as 'the banded mosquito of Bengal' from Kolkata, India. In laboratory conditions, many strains of A. albopictus have high-vector competence, but in natural conditions they are assumed to have low vectorial capacity since blood meals taken from nonsusceptible hosts do not contribute to the transmission cycle of CHIKV. However, the current epidemic of CHIKV confirmed that A. albopictus could have high vectorial capacity.

A recent study in Thailand showed evidence of an $A$. albopictus preference for human blood. A possible reason for this could be the relative abundance of human beings in the peridomestic environment, which could oblige the mosquito to feed on human beings. The vector $A$. albopictus has been introduced in several European countries: Albania, Belgium, Bosnia and Herzegovina, Croatia, France, Greece, Italy, The Netherlands, Serbia and Montenegro, Slovenia, Spain and Switzerland, mainly through the importation of tires and lucky bamboo (decorative plant), resulting in its establishment in most of Albania, northern/central Italy and limited areas within other countries. A. albopictus has been found to be active all year round in the tropical (e.g., Central America) and subtropical (e.g., the Gulf Coast) latitudes of America.

'Through a feral transmission cycle, the virus is maintained between mosquitoes and nonhuman primates, which may further aid transmission to humans.'

However, the conceivable role of other species, especially $A$. aegypti, cannot be ignored in the transmission cycle and spread of CHIKV in tropical Asia. Both Aedes species are widely distributed throughout India. In India, $A$. aegypti is the major vector that maintains the human-mosquito-human cycle. However, entomological surveys have revealed high densities of $A$. albopictus in parts of India. Although $A$. aegypt $i$ is considered a primary vector in India, the role of $A$. albopictus cannot be ruled out.

\section{An encounter before birth: transplacental (maternal-fetal) transmission of CHIKV}

Vertical maternal-fetal CHIKV transmission and severe congenital CHIKV disease have been observed in some cases in the Réunion Island. Based on virological evidence, a direct role of CHIKV in fetal deaths has been speculated. 
However, the pathogenic mechanism remains unknown. Vertical transmission in humans has been described previously in various other arboviral infections, but this is the first observation for the maternal-fetal transmission of CHIKV. CHIKV RNA was detected in the amniotic fluid and placenta. The presence of the viral genome in the placenta and amniotic fluid confirmed transplacental transmission of chikungunya. Persistence of CHIKV in the placenta confirmed that viral replication in that location is not sensitive to the maternal immune reaction. Elevated fever in the mother could also have proved lethal to the fetus.

'Acute infection with variable clinical features was common during the recent CHIKV outbreak.'

\section{Autochthonous/sustained transmissions from imported cases}

Imported cases of chikungunya have been reported in several European countries and the USA. The risks of autochthonous transmission from imported cases have been evaluated and at least one case has been reported in the current CHIKV pandemic. A nurse in France developed chikungunya fever (laboratory confirmed) 3 days after caring for a CHIKV-infected patient. Investigation of this case has revealed that there was a probable blood exposure incident. Previous incidents involving the transmission of CHIKV during laboratory procedures have been described. Viral transmission through direct contact with highly viremic blood appears to be the most plausible hypothesis to explain this autochthonous CHIKV infection. Direct transmission to healthcare workers cannot be ruled out in diseaseendemic countries, although this is difficult to identify.

\section{Is the pathogeny complex?}

Comprehensive and meticulous studies on the pathogenesis of the chikungunya fever are not currently available. It is believed that the infection cycle begins when the virion is taken up into cellular vesicles by receptor-mediated endocytosis. A pH drop caused by the merging of the carrier vesicle with a cellular lysosome digests nucleocapsid proteins and releases the genomic RNA to the cytosol where replication begins. The positive sense RNA is immediately translated. New virions spontaneously assemble following viral translation and replication. The virion then buds through cellular membranes.
Parasitized by mosquitoes, the virus is taken up by the host animal's blood. The virus replicates within the mosquito vector before it is introduced into a human host through a mosquito bite. The virus is introduced into the capillaries, and replicates in the vascular endothelium before spreading through the blood to other target tissues including the muscles, joints, skin and brain.

\section{Clinical manifestation}

Acute infection with variable clinical features was common during the recent CHIKV outbreak. Most patients with acute CHIKV infection presented with high fever, severe arthralgia and a rash lasting for a period of 1-7 days. The incubation period was usually 2-4 days. Body temperature often reached $38.5-40^{\circ} \mathrm{C}$, accompanied by intermittent chills. However, this acute phase lasts for only 2-4 days. The temperature may remit for 1-2 days, resulting in a 'saddle-back' fever curve. During the acute phase of CHIKV infection most patients experienced a headache, although this was not usually severe. The common symptoms of polyarthralgia are polyarticular pain predominantly affecting the small joints of the feet, hands, wrists and ankles, with less involvement of the larger joints. Pain was worse after getting up in the morning from bed and improved with mild exercise but was aggravated by strenuous exercise. In some patients, swelling also occurred at the joints but no fluid accumulation was observed. Patients with milder articular manifestations became symptom-free within a few weeks but more severe cases required several months. Generalized myalgia with back and shoulder pain was common. Cutaneous manifestations were typical with a flush over the face and trunk, followed by an itching maculopapular rash 5 days after onset. Conjunctival infection and sore throat have also been observed.

CHIKV infection has shown a somewhat different picture in younger patients. Arthralgia and arthritis occur but are less prominent and last for a shorter time. Rashes may be less frequent in infants and younger children but prominent flushing and the early appearance of maculopapular or urticarial eruption are better clinical symptoms of disease in younger patients. Along with common symptoms, mild hemorrhage, gastrointestinal complication, lymphadenopathy, vascular decompensation and cases of meningoencephalitis have been reported in many patients in Réunion. Neurovirulence (appearing clinically as meningoencephalitis) 
during CHIKV infection requires further mechanistic explanations. Severe thrombocytopenia and neutropenia complicated by septicemia have also been reported in a few patients. Those infected with the chikungunya are then immunized against the disease.

\section{Differential diagnosis}

Despite infecting millions of people worldwide, chikungunya infection has been neglected since its discovery. Worldwide, there are a number of other infections involving mosquito-transmitted viruses (arboviruses) with similar symptoms that may be confused with chikungunya, such as Sindbis, Ross River and dengue, as well as other viral, bacterial and parasitic infections with a similar clinical presentation (Box 2). These, together with a detailed travel history, should be considered in the differential diagnosis. Dual or multiple infections should also be looked at cautiously as some cases of dual infection with dengue and CHIKV in a single patient were reported during this outbreak.

\section{Laboratory scrutiny measures: known to few, useful to all}

Rapid diagnosis of CHIKV is necessary as a number of other infections mimic the clinical picture of chikungunya. The case definitions are listed in Box 3. Confirmatory diagnosis of CHIKV may be carried out by viral isolation and serology. The virus can be isolated from the blood or cerebrospinal fluid during the acute phase of illness by the inoculation of specimens to mosquito cell cultures or by inoculation into infant mice. Usually, the isolation of the virus is

\section{Box 2. Differential diagnosis of chikungunya.}

\section{Arboviruses}

- Dengue (often mistaken due to clinical similarities, especially in children, who may have hemorrhagic symptoms due to chikungunya virus [CHIKV]; and in cases of dual infections with dengue and CHIKV in a single patient), Sindbis and Ross River

\section{Viral diseases}

- Rubella and Parvovirus B19 (which cause arthralgia, particularly in women), other alphaviruses (which cause febrile polyarthralgia), mumps, herperviruses (Varicella zoster virus, Epstein-Barr virus and cytomegalovirus), measles, retroviruses (human T-lymphotropic virus-1, HIV) and hepatitis

\section{Bacterial diseases}

- Meningococcemia, scarlet fever and typhoid

\section{Parasitic diseases}

- Leptospirosis (also known as Weil's disease, canicola fever, canefield fever, nanukayami fever or 7-day fever) and malaria correlated with poor prognosis. The conventional serological tests are hemagglutination inhibition, complement fixation and neutralization. Demonstration of a rise in hemagglutination-inhibiton antibody titers between acute and convalescent serum samples is a widely used antibody detection method for diagnosis. Immunoglobulin $\mathrm{M}$ capture enzyme-linked immunosorbent assay has been used for the detection of CHIKV-specific IgM antibodies in the early phase of illness. A number of other sensitive tests described for the rapid diagnosis of CHIKV include immunofluorescence tests. Reverse transcriptase (RT)-PCR alone and RT-PCR in combination with nested PCR is generally used in the diagnosis of CHIKV.

\section{Predicting the chances of recovery: prognosis}

Symptoms are generally self-limiting and last 1-10 days. However, arthralgia may persist for several months or years. Most patients recover fully from CHIKV infection after a few days, although joint pain persists for a longer period after the other symptoms have disappeared. Although chikungunya is not considered to be fatal, some deaths have been reported to be associated with chikungunya on Réunion island.

\section{Treatment/management: where are we?}

There are no specific antiviral drugs against chikungunya, nor is there any vaccine against CHIKV. The situation suggests that despite infecting millions of people worldwide, the search for any antiviral against CHIKV has been neglected. Hence, treatment of chikungunya fever is still supportive only. Only rest, hydration, proper diet, movement and mild exercise and the combination of mild pain medications such as ibuprofen, naproxen, acetaminophen or paracetamol may relieve the symptoms of fever and aching, provided that the person has no contra-indications to these medications. Salicylate increases the risk of bleeding and possibly Reye syndrome, hence it should be avoided during the acute stages of the illness. Re-evaluation and closer monitoring is advised in chronic ailments.

Those already infected with chikungunya are then immunized against the disease. However, a promising vaccine candidate could be attenuated virus vaccine (TSI-GSD-218), developed in the 1980s by researchers at the US Army Medical Research Institute for Infectious Diseases. Clinical trials have revealed that this vaccine triggered neutralizing antibodies. 


\section{Box 3. Chikungunya case definition.}

\section{Suspected chikungunya diagnosis}

At least one of following:

- Cases with a sudden onset of high fever (ranging from $38.5-40^{\circ} \mathrm{C}$ ), muscle pain, headache, swelling and severe pain in the joints with polyarthralgia (pain in several joints) followed by an itching maculopapular rash

- Cases with any of the above symptoms together with a travel history to a chikungunya virus (CHIKV)-affected area

- Occurrence at the same location and time as confirmed cases of chikungunya fever

\section{Confirmed chikungunya diagnosis}

The above with at least one of the following laboratory confirmation of CHIKV infection:

- Detection of CHIKV nucleic acids (CHIKV-RNA) by reverse transcription-PCR

- Serological assays to screen for the anti-CHIKV-IgM antibodies (twofold rise in anti-CHIKV-IgM)

- Isolation and characterization of CHIKV

- Detection of CHIKV in tissue, serum or cerebrospinal fluid by immunohistochemistry, immunofluorescence or enzyme-linked immunosorbent assay on their sides when they are not being used. Such measures could represent the best preventive actions against the vector. Application of larvicidal treatment by larva-eating fish (Gambusia) or bacteria (Bacillus thuringiensis israelensis) should also be used to eliminate the vector. Fogging with pesticides, spraying of insecticides ( $2 \%$ pyrethrum) and the use of temephos larvicide in recommended doses could all be considered for the infected area. Awareness of the disease and vector, detection and elimination of vector breeding sources, proper facilities for healthcare and community awareness about the prophylactic measures are required to control the further spread of the disease.

'Long-term prevention policy should focus on the elimination of vectors and strategies for the development of specific antivirals and vaccines.'

\section{Possible strategies \& recommendations to combat CHIKV}

As a complex interplay of several factors has influenced CHIKV re-emergence, new policies and measures are required to avoid further escalation of the menace, which may include short- and long-term policies and strategies.

Short-term steps should focus on preventing the further spread of the problem, which should include instructing airline companies to provide an information sheet to all persons travelling to affected countries. Travellers who develop symptoms consistent with CHIKV infection should be recommended to consult a physician for follow-up and advice to avoid further spread. Public health authorities should make CHIKV fact sheets available to physicians. Healthcare staff should be reminded about the need to follow universally accepted precautions when handling samples from all patients, including patients presenting with chikungunya fever. In areas where Aedes mosquitoes are prevalent, and therefore where there is a higher risk of local transmission, public health authorities should provide clinical disease information to hospitals in order to raise their awareness about chikungunya fever as a differential diagnosis.

Long-term prevention policy should focus on the elimination of vectors and strategies for the development of specific antivirals and vaccines. In Aedes prevalent areas, its competence and capacity as a vector needs further investigation to assess the risk of dissemination and establishment of 
CHIKV. The identification of areas with consideration given to the climatic and ecological conditions favoring the establishment of the vector should be of prime concern, which could be achieved only by implementing or strengthening vector surveillance. Economic sanction should be considered as a measure for preventing the introduction of Aedes through trade (used tires and lucky bamboos). Although the disease is selflimiting, sustained and intensified control measures are required to control further spread of the disease worldwide. Mosquito control is the sole available method for reducing the transmission of chikungunya. Recently, in 2006 the United States Agency for International Development endorsed the indoor spraying of dichloro-diphenyl-ttrichloroethane and the WHO also supported it. It seems that a combination of strategies are needed to combat the menace. Collectively, wider issues of ecology, current agricultural practices, water management systems and human behavior patterns need to be investigated for policy decisions to be made.

\section{Concluding remarks}

The resurgence of CHIKV to such a great degree, and its wider epidemiological spread, is a major cause for concern. It suggests that any infectious agents can re-emerge with new efficient strains, even several years post-disappearance. For the first time, several deaths and the vertical maternal-fetal transmission of the virus has been observed in Réunion island, suggesting the re-emergence of a more virulent strain. The unprecedented magnitude of the epidemic also outlines the complete failure of vector control programs in affected countries. The lack of heard immunity in fast-growing populations and easier and faster dispersal of infectious agents have posed new challenges. Traveling has emerged as a potent cause of extensive and rapid dispersal of infectious agents to other countries, as several imported cases have been reported in Europe and America. In the absence of complete information on various aspects of CHIKV, early control appears to be difficult. Thus, there is an immediate need to initiate CHIKV research, which should include the development of effective antivirals and vaccine strategies. Special emphasis should be placed on strengthening vector surveillance and control programs and developing rapid diagnostic skills.

The current outbreak suggests a need for the establishment of a system for the continuous surveillance of emerging and re-emerging diseases. The re-emergence of such viral diseases is an unpredictable phenomena but the impact of such events can be mitigated by appropriate knowledge about the disease-causing agent and being prepared. Finally, measures to improve clinical management, especially in terms of early detection, nutritional support to affected patients and other preventive measures, may largely reduce disease. Considering the high number of cases and the lack of specific antiviral therapy, it is imperative to proceed with a sense of urgency in this matter.

\section{Bibliography}

1. Saxena SK, Singh M, Mishra N, Lakshmi V: Resurgence of chikungunya virus in India: an emerging threat. Euro Surveill. 11 E060810.2 (2006)

2. Schuffenecker I, Iteman I, Michault A et al.: Genome microevolution of chikungunya viruses causing the Indian Ocean outbreak. PLoS Med. 3, E263 (2006).
3. Briolant S, Garin D, Scaramozzino $\mathrm{N}$ et al: In vitro inhibition of chikungunya and semliki forest viruses replication by antiviral compounds: synergistic effect of interferon- $\alpha$ and ribavirin combination. Antiviral Res. 61, 111-117 (2004).

\section{Affiliation}

- Shailendra K Saxena, PhD, DCAP, FAEB, FCMS Centre for Cellular \& Molecular Biology, Uppal Road, Hyderabad 500007 (AP), India Tel.: +914027192630 (direct); +91402716 0222; ext. 2630; Fax: +914027160591 +914027160311; shailen@ccmb.res.in; shailen1@gmail.com 